\title{
Designing Response Surface Model-Based Run-by-Run Controllers: A Worst Case Approach
}

\author{
John S. Baras, Fellow, IEEE, and Nital S. Patel, Member, IEEE
}

\begin{abstract}
This paper presents a framework for carrying out run-by-run (RbR) control via a deterministic worst-case approach. In particular the $R b R$ controller developed tries to minimize the worst-case performance of the plant. This yields a methodology for handling uncertainty. A consequence of using the deterministic approach is that we no longer need any assumptions on the statistics of the noise. Rather, what we require is that the noise be bounded. Thus, we can also deal with nonGaussian and correlated noise. We provide results comparing the performance of the controller to a recursive least squares (RLS) based controller.
\end{abstract}

Index Terms - Run-by-run control, deterministic approach, bounded noise, worst-case design, ellipsoidal algorithms, response surface models.

\section{INTRODUCTION}

$\mathbf{R}^{\mathrm{s}}$ ECENTLY, there has been a strong interest in run-byrun (RbR) control in the semiconductor industry. With device tolerances shrinking, it becomes necessary to squeeze maximum performance out of existing equipment. A further advantage of the RbR control framework is that it enables automatic recipe generation to meet different targets, and also aids in the recovery of the process after a large disturbance. In this paper, we present a worst case framework for carrying out $\mathrm{RbR}$ control. The advantage of this approach is its capability to handle uncertainty. This is useful in cases when we do not have confidence in our models, such as after a sudden change in process characteristics. More importantly, the approach allows us to relax the assumptions placed on the noise statistics. Toward the end of this paper, we present an example where the controller successfully handles both correlated skewed Gaussian as well as correlated non-Gaussian noise.

As with any control strategy, some a priori information needs to be available about the process model. What we require is the structure of the map between the recipe and the measured variables. Such maps could be provided by models obtained via response surface methodology (RSM). However, the conceptual development is not restricted to RSM alone. A number of researchers have successfully employed RSM to the

Manuscript received February 12, 1996; revised June 1996. This work was supported by the National Science Foundation Engineering Research Centers Program: NSFD CDR 8803012 and the Lockheed Martin Chair in Systems Engineering. This paper was presented in part at the 1995 IEEE/CPMT International Electronics Manufacturing Technology Symposium, October 2-4, 1995, Austin, TX.

The authors are with the Institute for Systems Research and Department of Electrical Engineering, University of Maryland, College Park, MD 20742 USA.

Publisher Item Identifier S 1083-4400(96)06096-2. problem of automated recipe generation, process optimization and design, [1]-[7].

In addition to the above, we will also need bounds on the process noise, in the sense that the assumed model, with these noise bounds can account for $(1-\alpha) \%$ of the observations, where $\alpha$ is a very small number. For example, if we choose $\alpha$ to be 0.27 , then the model with these noise bounds can account for $99.73 \%$ of the observations. The problem of selecting these bounds is similar to the problem of specifying control limits for control charts in statistical process control (SPC). The idea here is that if we define our process model in this manner, then the RbR controller hardly ever observes process results inconsistent with the model, and if the results are in fact inconsistent, they would also generate an alarm via SPC. As will be clarified, by carrying out consistency checks on every measurement, the RbR controller can also generate alarms. The influence of these bounds on the performance of the RbR controller is similar to that observed in control charts. For example, if the bounds are chosen to be smaller than what they actually are, the controller will generate an alarm, even if the process is in control. On the other hand if they are too lax, then the controller becomes less sensitive to process variation. For purposes of the controller design, what is of importance are the noise bounds, and not the actual noise statistics. In particular, one maybe able to obtain good bounds without any deep statistical considerations. Fig. 1 aims to graphically display this concept (for purposes of clarity, the quantity has been chosen to be vector valued). The dark dot is the model prediction, and the other dots are the measurements for a fixed recipe setting. One can either account for this scatter via statistical considerations, or by placing bounds on their magnitude. In the latter case, of course one has the possibility of occurrence of outliers. The idea is to choose the bounds so as to make their occurrence as remote as possible.

These bounds are linked to the basic process variance (process noise), over which we have no control and the prediction error of the model (which need not have a polynomial structure). For example, if we assume that both the model error, and the process noise are independently normally distributed with (in the single measurement case) variances $\sigma_{M}^{2}$, and $\sigma_{P}^{2}$, respectively, then the bounds around the model are $\pm 3 \sqrt{\sigma_{P}^{2}+\sigma_{M}^{2}}$ for $\alpha=0.23$. An interesting problem in this regard is given the upper and lower control limits of a variable, and the process noise statistics, what order model is required to ensure that the combined model prediction error and the process noise will still result in $(1-\alpha) \%$ of the 


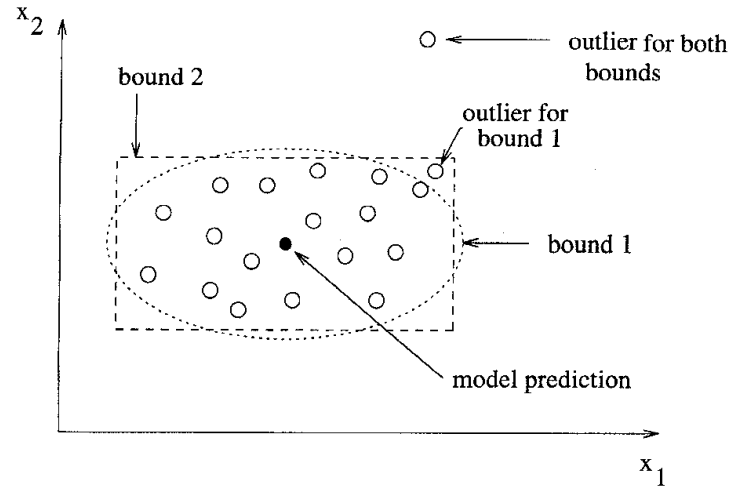

Fig. 1. Plot of model prediction and actual measurements for a vector valued quantity. One can account for the scatter either by statistical considerations, or by placing noise bounds. Two such bounds and their associated outliers are shown above.

observations falling between the control limits. Furthermore, for quick recipe calculation, we need the smallest number of terms in the model as possible. Initially, one can determine this via a designed experiment. However, once the RbR controller is implemented the process noise statistics and/or the error statistics of the model may change. Currently, work is underway to implement a strategy for carrying out automated online modifications of the model structure.

The rest of this paper is organized as follows. The general conceptual framework is presented in Section II. Section III specializes the framework to polynomial models. This is followed by simulation results in Section IV and conclusions in Section V.

\section{CONCEPTUAL FRAMEWORK}

This section gives the general framework under which we develop the RbR controller. The system is modeled as

$$
\begin{aligned}
M_{k+1} & \in \mathcal{F}\left(M_{k}\right), \quad M_{0}=\bar{M} \\
y_{k+1} & \in \mathcal{G}\left(M_{k}, u_{k}\right) \\
z_{k+1} & =l\left(y_{k+1}\right), \quad k=0, \cdots .
\end{aligned}
$$

Here, $y_{k} \in \boldsymbol{R}^{t}$ are the variables to be controlled, $z_{k} \in \boldsymbol{R}$ is the cost which is a function of $y_{k}, u_{k} \in U \subset \boldsymbol{R}^{m}$ is the vector of recipes, and $M_{k} \in \mathcal{M}$ are disturbance driven states. At this stage, the structure of $\mathcal{G}, \mathcal{F}, l$, and $M_{k}$ has been left undefined, since the conceptual framework is applicable to any such structure. In the next section, we will fix the structure to obtain an implementable solution. Furthermore, note that the recipes are assumed to belong to the set $\boldsymbol{U}$. One could consider $\boldsymbol{U} \equiv \boldsymbol{R}^{m}$, however, in practice this will hardly ever be the case. In fact, natural bounds can be placed on $\boldsymbol{U}$ based on the operating limits of the equipment, using engineering judgment, or by forcing $\boldsymbol{U}$ to be the set of recipes over which the model is valid. To avoid confusion, the run numbers will be indexed beginning with one. Hence, the inputs and measurements for the $j$ th run are $u_{j-1}$ and $y_{j}$, respectively.
The aim of the RbR is to minimize the worst case cost on the onset of every run. Although, the cost has been assumed to be scalar, we could have considered a multi-objective problem with the aim of obtaining Pareto-optimal recipes. We could have also penalized changes in the recipe settings by incorporating additional terms in the cost function $l$, or restricted the maximum allowable change by redefining $U$ at the onset of each run as a function of the previous recipe settings. The only change required would be in the final optimization stage [defined later on in (4)].

At this time, it becomes necessary to differentiate between what we call i) nominal disturbances and ii) exceptional disturbances. Nominal disturbances represent the changes that the process normally undergoes between runs. An example of this is drift due to equipment aging. We assume that one can bound these and represent them via $\mathcal{F}$. Exceptional disturbances on the other hand, are those that are not represented by our model (1). Their magnitude is, in general, much larger than that of the nominal disturbances, and they occur infrequently. They can be caused by various reasons, such as maintainence operations, e.g., renewal of parts. Furthermore, exceptional disturbances will also flag an error in the consistency check carried out by the RbR controller. The process noise is modeled separately from the above mentioned disturbances, and is included in $\mathcal{G}$.

We now turn to the problem of designing the RbR controller for the system (1). Assuming we have carried out $j$ runs, we have available to us measurements $y_{k}$ for $k=1, \cdots, j$, and past recipes $u_{k}$ for $k=0, \cdots, j-1$. Based on this, we compute the set of feasible states $\boldsymbol{P}_{j}$ that the system could be in during run $j+1$ assuming that we will not encounter an exceptional disturbance. We can carry out this computation recursively as follows: First compute

$$
\mathcal{P}_{j}=\left\{M \in P_{j-1}: y_{j} \in \mathcal{G}\left(M, u_{j-1}\right)\right\}
$$

and then calculate $P_{j}$ as

$$
\boldsymbol{P}_{j}=\bigcup_{M \in \mathcal{P}_{j}} \mathcal{F}(M)
$$

The initial set $\boldsymbol{P}_{0}$ could be defined depending on the amount of confidence one has on the initial value of the states $\bar{M}$. Once, we obtain $\boldsymbol{P}_{j}$, we obtain the recipe $u_{j}^{*}$ which solves

$$
\min _{u \in \boldsymbol{U}} \max _{M \in \boldsymbol{P}_{j}} \max _{y \in \mathcal{G}(M, u)} l(y)
$$

In the development above, we have ignored the special case when the set $\mathcal{P}_{j}$ calculated in (2) is empty. In this case, the problem is similar to that encountered in SPC, i.e., to determine whether an exceptional disturbance occurred, or that $y_{j}$ is just a bad data point. This situation can be handled in various ways. Some of these are as follows:

1) Assume that an exceptional disturbance occurred and reset $\boldsymbol{P}_{j-1}=\mathcal{M}$. Now recompute $\mathcal{P}_{j}$. In the next section, and during simulations, we adopt this approach. 
2) Assume that $y_{j}$ is a bad data point. In this case, set $\mathcal{P}_{j}=\boldsymbol{P}_{j-1}$. If the process has shifted, this strategy will generate a large number of faults. We check the number of faults generated in a fixed number of runs, and if this exceeds some threshold value, we reset the set of feasible states to $\mathcal{M}$.

3) One could also assume that either of the two cases mentioned above occurred. That is, the process could have undergone an exceptional shift, or $y_{j}$ is a bad data point. In such a case, we first set $\hat{\mathcal{P}}_{j}=\boldsymbol{P}_{j-1}$ (i.e., assume $y_{j}$ is a bad data point), and then set $\boldsymbol{P}_{j-1}=\mathcal{M}$, recompute $\mathcal{P}_{j}$, and set $\overline{\mathcal{P}}_{j}=\mathcal{P}_{j}$ (i.e., assume an exceptional shift occurred). Then, we redefine $\mathcal{P}_{j}=\overline{\mathcal{P}}_{j} \cup \hat{\mathcal{P}}_{j}$

If $\mathcal{F}(M)=\{M\}$, i.e., one does not expect the process to change between runs, then

$$
P_{j} \subset P_{j-1}
$$

and, hence, we have a sequence of nested sets. However, if $\mathcal{F}(M)$ is a set, we can no longer guarantee that the sets remain nested. However, informally one may argue that the larger $\boldsymbol{P}_{j}$ becomes, more of its elements are invalidated by the next observation $y_{j+1}$ and, hence, the sequence of sets $\boldsymbol{P}_{j}, j=0,1, \cdots$ will ultimately be bounded. Moreover, since for normal operation, $\mathcal{F}(M)$ will be a small set, the inflation introduced by it will also be small. While carrying out simulations, we have observed that this is indeed the case, and the sets $\boldsymbol{P}_{j}$ do in fact remain bounded. Work is currently in progress to derive analytic bounds on these sets.

The main difficulty in the general approach is the excessive computational time required to calculate $P_{j}$ in (3), and for solving the optimization problem with respect to $\boldsymbol{P}_{j}$ in (4). However, this result may be used off-line to estimate the best guaranteeable performance achievable under the given model assumptions. We now turn to a technique for approximating these sets using ellipsoids. However, before doing so, we need to impose a suitable structure on the models.

\section{POLYNOMIAL MODELS AND ELLIPSOIDAL APPROXIMATIONS}

Considerable simplifications can be obtained in the above developments, if we impose a polynomial structure on the models. An additional advantage is that this is compatible with the models obtained via RSM [8]. In general, since these are obtained for one quantity at a time, we treat the problem of having $t$ outputs as, $t$ single output problems in parallel. For the $i$ th output $y_{i}$, we model $\mathcal{G}_{i}\left(\theta_{i}, u\right)$ as an ellipsoid given by

$$
\mathcal{G}_{i}\left(\theta_{i}, u\right) \triangleq\left\{y \in R: g_{i}^{-1}\left(y-\theta_{i}^{T} u\right)^{2} \leq 1\right\}
$$

where $u=\left[\begin{array}{lll}1 & v_{1} & v_{2} \cdots v_{m}\end{array}\right]^{T}$, with $v_{j}, j=1, \cdots, m$ being known functions of the recipe settings, and $\theta_{i}$ being the vector of coefficients. In particular, $\theta_{i}^{T} u$ represents the response surface for the quantity $y_{i}$, and $g_{i}>0$ represents the bound on the noise. Also, (5) implies that

$$
y_{i} \in\left[\theta_{i}^{T} u-\sqrt{g_{i}}, \theta_{i}^{T} u+\sqrt{g_{i}}\right] .
$$

This representation can take care of nonsymmetric bounds on the noise by adding a bias term to the first element of $\theta_{i}$.

We now turn to the definition of $\mathcal{F}_{i}(\theta, u)$ which we also define as an ellipsoid as

$$
\mathcal{F}_{i}(\bar{\theta}) \triangleq\left\{\theta \in R^{m}:(\theta-\bar{\theta})^{T} F_{i}^{-1}(\theta-\bar{\theta}) \leq 1\right\}
$$

with $F_{i} \in R^{m \times m}, F_{i}>0$, i.e., $F_{i}$ is a positive definite matrix. As an example, for the case of a process with drift only, $F_{i}$ will be diagonal with very small entries for all the diagonal elements, except the first.

Ellipsoidal algorithms return an ellipsoidal estimate of the feasible parameter set. Specifically, on the onset of run $k+1$, we have

$$
\boldsymbol{P}_{i, k}=\left\{\theta_{i} \in \boldsymbol{R}^{m}:\left(\theta_{i}-\bar{\theta}_{i, k}\right)^{T} P_{i, k}^{-1}\left(\theta_{i}-\bar{\theta}_{i, k}\right) \leq 1\right\}
$$

where $\bar{\theta}_{i, k}$ is the center of the ellipsoid, and the matrix $P_{i, k} \in \boldsymbol{R}^{m \times m}, P_{i, k}>0$ specifies the size and orientation of the ellipsoid. Various ellipsoidal algorithms exist in the literature, [9]-[11]. The algorithm implemented by us, has two stages, as follows:

1) First using the optimal volume ellipsoid (OVE) approach of $[10]$, we try to find the minimum volume ellipsoid $\hat{\boldsymbol{P}}_{i, k}$ which bounds the set

$$
\left\{\theta_{i} \in P_{i, k-1}: y_{k} \in \mathcal{G}_{i}\left(\theta, u_{k-1}\right)\right\}
$$

where $P_{i, k-1}$ is the ellipsoidal estimate of $\theta_{i}$ at the onset of run $k$.

2) We then inflate $\hat{\boldsymbol{P}}_{i, k}$ by finding the minimal volume ellipsoid which bounds

$$
\bigcup_{\theta \in \hat{\boldsymbol{P}}_{i, k}} \mathcal{F}_{i}(\theta)
$$

and set that equal to $\boldsymbol{P}_{i, k}$. For this stage, we use the result from [12].

The initial values of $P_{i, 0}$ can be fixed depending on the amount of confidence we have on the initial parameter vectors $\bar{\theta}_{i, 0}$. Furthermore, if the intersection in Stage 1) of the update algorithm returns an empty set, we reset the entries of $P_{i, k-1}$ to large values (in certain cases, we may be able to use our judgment and only modify selected entries) and then repeat Stage 1).

Now, assuming we have $t$ outputs and, hence, $t$ ellipsoids characterized by $\left(\bar{\theta}_{i, k}, P_{i, k}\right), i=1, \cdots, t$, we can pose the 
final optimization problem (4) as

$$
\min _{u \in \tilde{U}} \max _{\underline{y}_{k} \leq y \leq \bar{y}_{k}} l(y)
$$

where $\hat{\boldsymbol{U}}=\left\{\left[u_{0} v\right]^{T}: u_{0}=1, v \in \boldsymbol{U}\right\}$, and $\underline{y}_{k}, \bar{y}_{k}$ are $t$ dimensional vectors, whose $i$ th components are given by $\bar{\theta}_{i, k}^{T} u-\sqrt{u^{T} P_{i, k} u}-\sqrt{g_{i}}$ and $\bar{\theta}_{i, k}^{T} u+\sqrt{u^{T} P_{i, k} u}+\sqrt{g_{i}}$, respectively. We now force $l$ to be convex with respect to $y$ (such as a quadratic cost function). Then, the inner maximization is achieved at one of the vertices of the box defined by $\underline{y}_{k}, \bar{y}_{k}$ and the optimization algorithm takes advantage of this fact to cut down the complexity.

We mention here the fact, that the OVE algorithm does not propagate the center of the ellipsoid as a weighed recursive least squares (WRLS) estimate. However, there do exist ellipsoidal algorithms which do propagate the center as a WRLS estimate of the model parameters [9], [13]. In these cases, however, the ellipsoid may greatly overbound the feasible set and, hence, could yield very conservative recipes. If, however, the bound is tight, then one can view (7) as minimizing the cost based on the WRLS estimate with modulation due to the uncertainty in the estimate. However, trying to measure the tightness of the bounds is as hard as solving for the actual feasible set, i.e., the exact problem of the previous section.

Note that since the feasible set for each output is computed independently of the others, we could sample the different outputs at different rates with only minor modifications to the structure of the $\mathrm{RbR}$ controller. For example, if we had only two outputs $\left(y_{1}, y_{2}\right)$, we could sample $y_{1}$ after every run, and $y_{2}$ after every two runs. Then we would update $\boldsymbol{P}_{1}$ as mentioned above. However, for $\boldsymbol{P}_{2}$, we would do a full update only when we obtain a new measurement, else we will only inflate it as done in Step 2) of the update algorithm. This also raises the possibilities of carrying out multi-rate sampling.

\section{Simulation Results}

In this section, we present some simulation results. We consider four cases: i) system under steady drift, ii) a step disturbance, iii) presence of bad data points, and iv) correlated non-Gaussian noise. Although, the first three cases assume a normal distribution of the underlying noise, the distribution as seen by the controller is in fact skewed due to a nonlinear (ln) transformation of the data. The simulations are based on the models for an LPCVD reactor presented in [14]. Here, we limit our concern to the deposition rates on the first and last wafer. We augment the models with drift terms. The models express the deposition rates in terms of deposition temperature $T$, deposition pressure $P$, and the silane flow rate $Q$. They are given by

$$
\begin{aligned}
& \hat{R}_{1}=\exp \left(c_{1}+c_{2} \ln P+c_{3} T^{-1}+c_{4} Q^{-1}\right)+d_{1} \\
& \hat{R}_{2}=\hat{R}_{1}\left[\frac{1-S^{\prime} C_{g s} \hat{R}_{1} Q^{-1}}{1+S^{\prime} C_{g s} \hat{R}_{1} Q^{-1}}\right]+d_{2}
\end{aligned}
$$

with the rates expressed in $\AA / \mathrm{min}, P$ in mtorr, $T$ in $\mathrm{K}$, and $Q$ in sccm. The parameters are given [14] to be $c_{1}=20.65$, $c_{2}=0.29, c_{3}=-15189.21, c_{4}=-47.97, S^{\prime}=4777.8$, and $C_{g s}=1.85 \times 10^{-5}$, where we have clropped the units for convenience. $d_{1}$, and $d_{2}$ represent the drift terms. The measured rates are obtained from the above model by adding a zero mean noise to $\hat{R}_{1}$ and $\hat{R}_{2}$. For the first two cases, we take it to be Gaussian with variance nine, and for the last case, we filter it to obtain a colored noise. Furthermore, the maximum drift expected between runs is 0.3 . This actually represents a shift of $\sigma$ in 10 runs, and may be too large to be true in practice. However, we choose this value, since it enables us to see the corrective action of the RbR controller in a fewer number of runs. The targets $Y_{1}$ for $R_{1}$, and $Y_{2}$ for $R_{2}$ are fixed at $169.75 \AA / \mathrm{min}$ and $141.7 \AA / \mathrm{min}$, respectively.

For the purpose of the RbR controller, we work in the In space. The controller observes $y_{1}=\ln R_{1}$, and $y_{2}=\ln R_{2}$. We now set $u_{1}=\ln P, u_{2}=T^{-1}$, and $u_{3}=Q^{-1}$, and define the vector $u=\left[\begin{array}{llll}1 & u_{1} & u_{2} & u_{3}\end{array}\right]^{T}$. Assuming, that the RbR controller keeps the process on target, we fix $g_{1}$ and $g_{2}$ as 0.0025 . Note, that this value yields smaller bounds than the actual bounds on the noise, however, due to overbounding by the ellipsoids, no consistency error was generated by the RbR controller. Based on the drift information, we now fix $F_{1}$ and $F_{2}$ as

$$
\begin{aligned}
& F_{1}=\left[\begin{array}{cccc}
3.2 \times 10^{-4} & 0 & 0 & 0 \\
0 & 10^{-12} & 0 & 0 \\
0 & 0 & 10^{-12} & 0 \\
0 & 0 & 0 & 10^{-12}
\end{array}\right] \\
& F_{2}=\left[\begin{array}{cccc}
4.7 \times 10^{-4} & 0 & 0 & 0 \\
0 & 10^{-12} & 0 & 0 \\
0 & 0 & 10^{-12} & 0 \\
0 & 0 & 0 & 10^{-12}
\end{array}\right] .
\end{aligned}
$$

The initial parameter vectors $\theta_{1,0}$, and $\theta_{2,0}$ are fixed as

$$
\theta_{1,0}=\left[\begin{array}{c}
20.65 \\
0.29 \\
-15189.21 \\
-47.97
\end{array}\right] ; \quad \theta_{2,0}=\left[\begin{array}{c}
16.3509 \\
0.2177 \\
-10992 \\
-71.774
\end{array}\right]
$$

where $\theta_{2,0}$ is obtained by fitting a polynomial model to the data generated by the system (8). The model is valid for a fixed range of the inputs, namely, the experimental design space. Hence, we place bounds on the recipe settings, and these translate to the vector $u$ as follows: $5.67 \leq u_{1} \leq 6.33$, $1.053 \times 10^{-3} \leq u_{2} \leq 1.1777 \times 10^{-3}$, and $0.003 \leq u_{3} \leq 0.01$. We initialize $P_{1,0}=P_{2,0}=10^{-12} I$, where $I$ is a $4 \times 4$ identity matrix. Finally, the cost is expressed as a quadratic function of the measurements as

$$
l(y)=w_{1}\left(y_{1}-\ln Y_{1}\right)^{2}+w_{2}\left(y_{2}-\ln Y_{2}\right)^{2}
$$

where $w_{1}=\left(\ln Y_{1}\right)^{2}$ and $w_{2}=\left(\ln Y_{2}\right)^{2}$. These, weigh the component involving $y_{1}$ more than that involving $y_{2}$, since the former is less sensitive to error in the deposition rate, due 

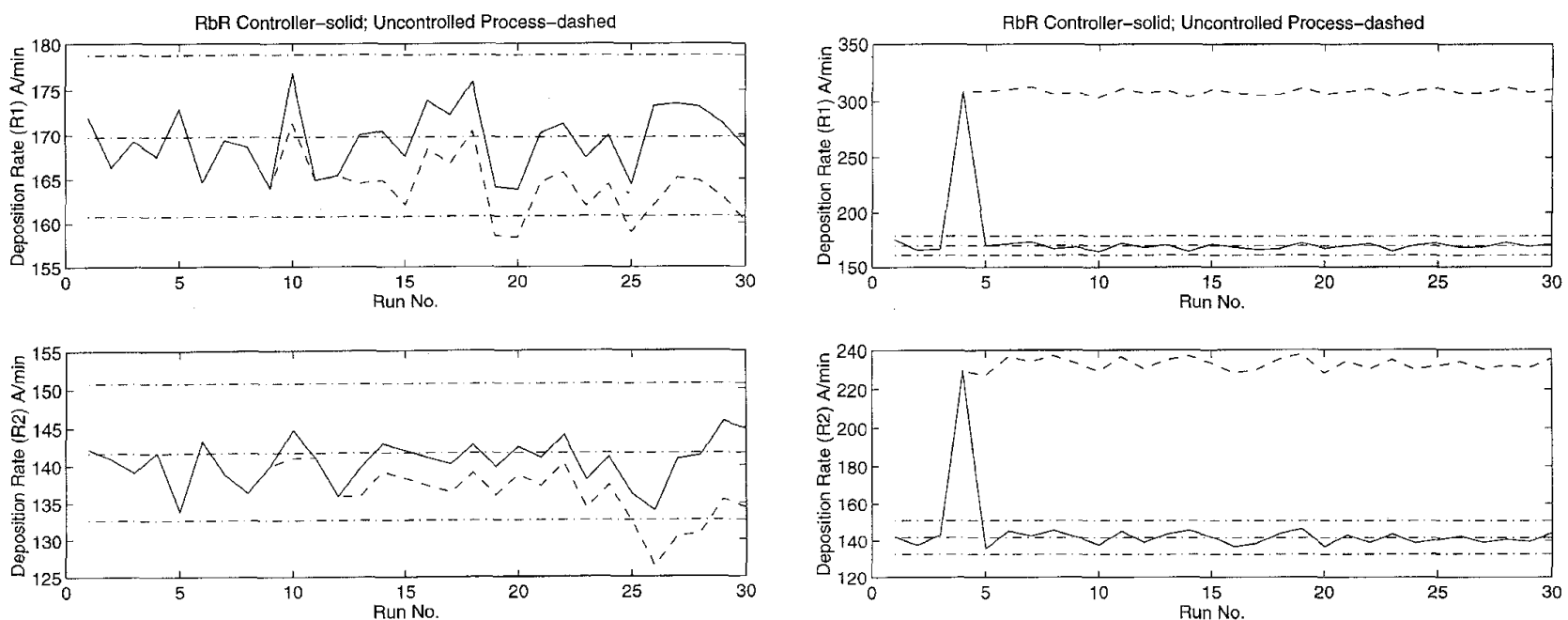

Fig. 2. Process under drift.

Fig. 3. Process subject to a step disturbance.

to the nonlinearity of the $\ln$ transformation. Moreover, one can experiment with different weights, however, our results show these weights to be good enough.

\section{A. Drifting Process}

We let both the equations (8) have a drift of $-0.3 \AA / \mathrm{min}$ between runs. Simulation results for both the controlled and uncontrolled trajectories are displayed in Fig. 2. The dash-dot lines give the target and the $3 \sigma$ noise bounds, where $\sigma=3$ is the standard deviation of the noise. For comparison, both the controlled and uncontrolled trajectories have been obtained under identical noise conditions. The conservative nature of the controller is apparent by the fact that the corrections are carried out infrequently.

\section{B. Step Disturbance}

Here, we change the process parameters after the third run. The parameters are changed to $c_{3}=-14600, c_{4}=-55.97$, $d_{2}=11$, and $C_{g s}=1.57 \times 10^{-5}$. The controller generated a consistency fault after run three, and we reset $P_{1}$ and $P_{2}$ to $P_{1,3}=P_{2,3}=10^{6} \mathrm{I}$. Fig. 3 shows the controlled and uncontrolled trajectories obtained under identical noise conditions. The dash-dot lines represent the target, and the noise limits as in Fig. 2. the controller corrects for the shift in the very next run and then leaves the process alone (as can be seen by comparing the controlled and uncontrolled trajectories).

\section{Bad Data Points}

Two bad data points are generated during the simulation runs. These occur during run 10 for $R_{1}$, and run 20 for $R_{2}$. The controlled trajectories are shown in Fig. 4. The dash-dot lines represent the target, and the noise limits. The simulations show the controller to be minimally affected by bad data points.
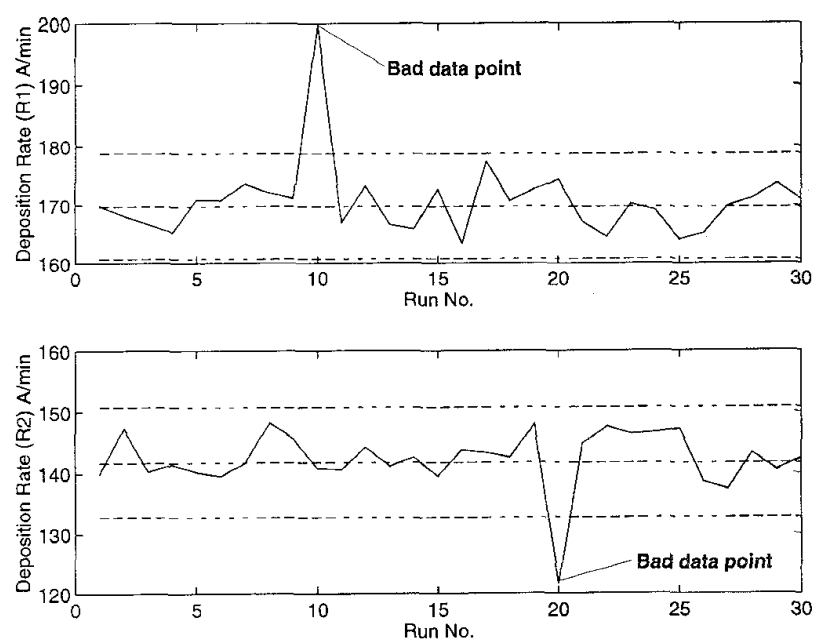

Fig. 4. Effect of bad data points.

\section{Skewed, Correlated, and Non-Gaussian Noise}

It should be noted that the noise seen by the controller is in fact skewed due to the $\ln$ transformation of the data. We now color the noise via filters, such that the first correlation coefficient (i.e., $\left.E\left(n_{i} n_{i-1}\right) / \sigma^{2}\right)$ is equal to -0.2680 for the noise added to $\hat{R}_{1}$, and -0.0627 for the noise added to $\hat{R}_{2}$. The simulation is similar to the step disturbance case, and we present the plot for data from runs 6-30 in Fig. 5. Here, WCA corresponds the controller designed in this paper. We also plot the output (shown via a dashed line) obtained by using a controller based on the recursive least squares (RLS) estimate of the model coefficients. For purposes of comparison, both the WCA and RLS-based controllers were simulated with the process subject to identical noise.

Finally, we present results for non-Gaussian noise. Note that the development of the controller does not assume anything about the noise statistics. All that is required is that the bounds 

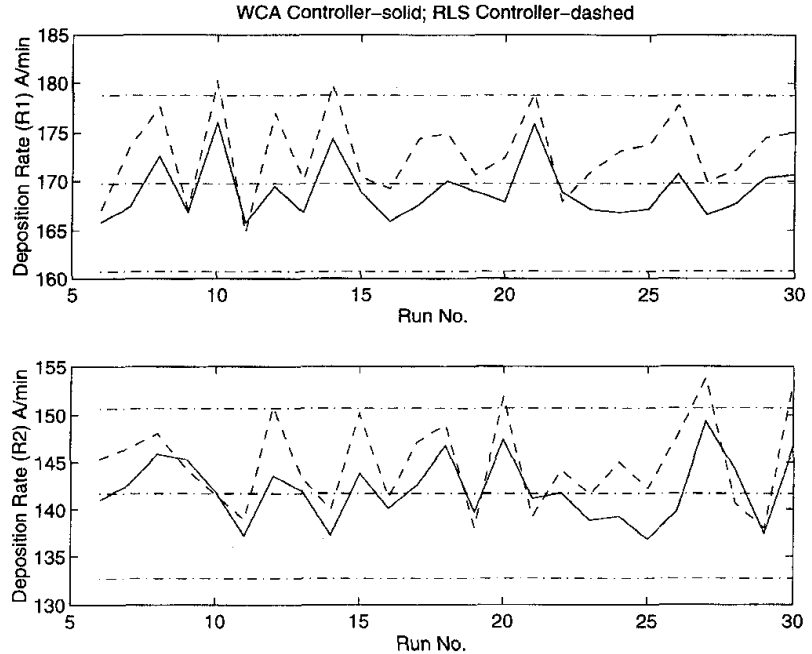

Fig. 5. Process subject correlated noise.
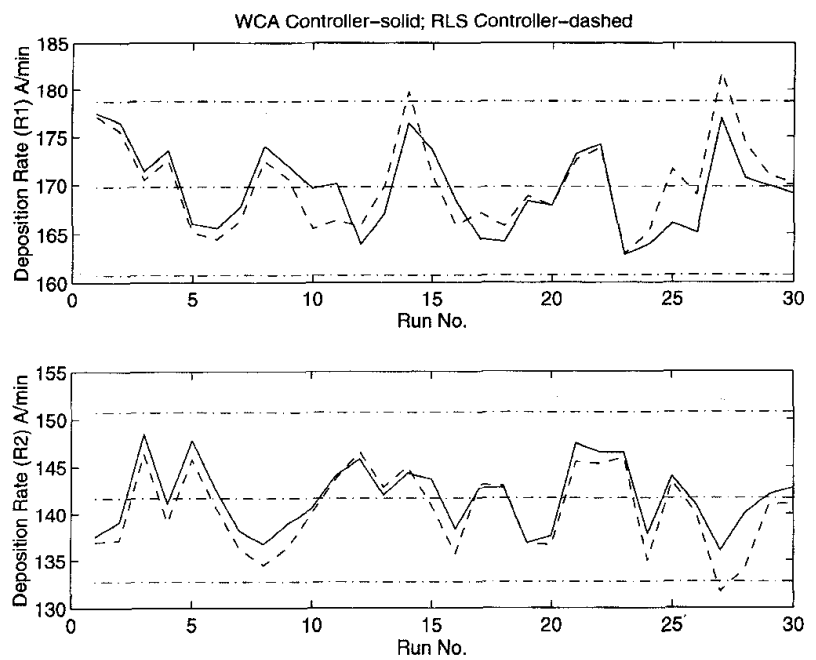

Fig. 6. Process subject to non-Gaussian correlated noise and steady drift.

on the noise be well chosen. For the purposes of generating the noise, we employ a uniform distribution on the interval $[-8,8]$, and a normal distribution with mean zero and standard deviation 0.33 . The noise value added to the model rates are obtained by adding together two random variables whose values are generated according to the uniform and normal distributions, respectively. These are then passed through a filter to yield correlated noise with the first correlation coefficient of 0.34 for both the noise inputs (i.e., to $\hat{R}_{1}$ and $\hat{R}_{2}$ ). The results for this case with the system subject to a steady drift of $-0.3 \AA / \mathrm{min}$ are presented in Fig. 6. Again, the dashed line is the trajectory obtained by employing a least squares estimate-based controller.

The means and standard deviations (STD) of the outputs for both the cases are given in Table I. The RbR controller presented in this paper outperforms the RLS-based controller in terms of both mean and standard deviation.
TABLE I

COMPARISON OF WCA AND RLS CONTROLLERS

\begin{tabular}{l|l|lr||lr}
\hline \hline \multicolumn{2}{l|}{} & \multicolumn{3}{|l|}{ Skewed/Correlated } & Non-normal/Correlated/Drift \\
\hline \multirow{2}{*}{ RLS } & $R_{1}$ & mean & 172.90 & mean & 170.02 \\
\cline { 3 - 6 } & & STD & 4.19 & STD & 4.61 \\
\cline { 3 - 6 } & $R_{2}$ & mean & 144.87 & mean & 140.33 \\
\cline { 3 - 6 } & & STD & 4.81 & STD & 4.30 \\
\hline \multirow{2}{*}{ WCA } & $R_{1}$ & mean & 169.04 & mean & 169.66 \\
\cline { 3 - 6 } & & STD & 2.96 & STD & 4.35 \\
\cline { 3 - 6 } & \multirow{2}{*}{$R_{2}$} & mean & 142.08 & mean & 141.77 \\
\cline { 3 - 6 } & & STD & 3.48 & STD & 3.60 \\
\hline \hline
\end{tabular}

\section{CONCLUSION}

A worst-case approach to RbR control is presented, and we have demonstrated its viability via simulation results. It is able to compensate for drifts, step disturbances, and is robust to bad data points. Furthermore, the method enables one to deal with arbitrary noise statistics.

Work is continuing to develop an expert system based monitor for automated model order changes, and to carry out online tuning of the RbR controller. We are also looking into an application of the controller to an industrial process.

We would also like to point out the fact that level sets in probability obtained from multivariate normal distributions are, in fact, ellipsoids. Hence, the ellipsoidal algorithm maybe equivalent to fitting a normal distribution to the statistics of the estimated process parameters. However, the exact relationship between the two is still an open question. One can also view the ellipsoids as estimating a confidence set for the model parameters.

\section{REFERENCES}

[1] S. Leang and C. J. Spanos, "Statistically based feedback control of photoresist application," in Proc. IEEE/SEMI Advanced Semicond. Manufact. Conf., 1991, pp. 185-190.

[2] S. Pan, M. T. Reilly, E. Di Fabrizio, Q. Leonard, J. W. Taylor, and F, Cerrina, "An optization design method for chemically amplified resist process control," IEEE Trans. Semicond. Manufact., vol. 7, no. 3, pp. $325-332,1994$.

[3] P. K. Mozumder and G. G. Barna, "Statistical feedback control of a plasma etch process," IEEE Trans. Semicond. Manufact, vol. 7, no. 1, pp. 1-11, 1994.

[4] S. Leang and C. J. Spanos, "Application of feed-forward control to a lithography stepper," in Proc. IEEE/SEMI Int. Semicond. Manufact. Science Symp., 1992, pp. 79-84.

[5] J. A. Power, B. Donnellan, A. Mathewson, and W. A. Lane, "Relating statistical MOSFET model parameter variabilities to IC manufacturing process fluctuations enabling realistic worst case design," IEEE Trans. Semicond. Manufact., vol. 7, no. 3, pp. 306-318, 1994.

[6] G. J. Gaston and A. J. Walton, "An integration of simulation and response surface methodology for the optimization of IC processes," IEEE Trans. Semicond. Manufact., vol. 7, no. 1, pp. 22-33, 1994.

[7] S. W. Butler and J. A. Stefani, "Supervisory run-to-run control of polysilicon gate etch using in situ ellipsometry," IEEE Trans. Semicond. Manufact., vol. 7, no. 2, pp. 193-201, 1994.

[8] G. E. P. Box and N. R. Darper, Empirical Model-Building and Response Surfaces. New York: Wiley, 1987.

[9] E. Fogel and Y. F. Huang, "On the value of intormation in system identification-Bounded noise case," Automatica, vol. 18, no. 2, pp. 229-238, 1982

[10] M. F. Cheung, S. Yurkovich, and K. M. Passino, "An optimal volume ellipsoid algorithm for parameter set estimation," in Proc. 30th IEEE Conf. Decision Contr., 1991, pp. 969-974. 
[11] F. C. Schweppe, "Recursive state estimation: Unknown but bounded errors and system inputs," IEEE Trans. Automat. Contr., vol. 13, no. 1, pp. 22-28, 1968.

[12] F. L. Chernous'ko, "Optimal guaranteed estimates of indeterminacies with the aid of ellipsoids," Engineering Cybernetics, vol. 18, pp. 1-9, 1980.

[13] J. R. Deller, M. Nayeri, and S. F. Odeh, "Least-square identification with error bounds for real time signal processing and control," Proc. IEEE, vol. 18 , no. 6, pp. $815-849,1993$.

[14] K.-K. Lin and C. J. Spanos, "Statistical equipment modeling for VLSI manufacturing: An application for LPCVD," IEEE Trans. Semicond. Manufact., vol. 3, no. 4, pp. 216-229, 1990.

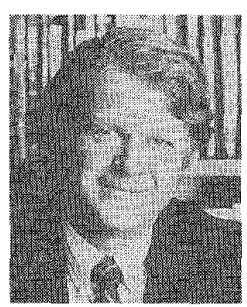

John S. Baras (S'73-M'73-SM' 83-F' 84) was born in Piraeus, Greece, on March 13, 1948. He received the B.S. degree in electrical engineering (with highest distinction) from the National Technical University of Athens, Greece, in 1970. He received the M.S. and Ph.D. degrees from Harvard University, Cambridge, MA, both in applied mathematics, in 1971 and 1973 , respectively.

Since 1973, he has been with the Department of Electrical Engineering, University of Maryland, College Park, where he is currently a Professor and member of the Applied Mathematics Faculty. From 1985 to 1991 , he was the Founding Director of the Systems Research Center, now the Institute for Systems Research. In February 1990, he was appointed to the Lockheed Martin Chair in Systems Engineering. Since 1991, he has been the Director of the Center for Satellite and Hybrid Communication Networks, a National Aeronautics and Space Administration (NASA) Center for the Commercial Development of Space, which he co-founded. He has held visiting research scholar positions at Stanford University, the Massachusetts Institute of Technology (MIT), Harvard University, the Institute National de Reserche en Informatique et en Automatique, and the University of California, Berkeley. His current research interests include stochastic systems, signal processing and understanding with emphasis on speech and image signals, real-time architectures, symbolic computation, intelligent control systems, robust nonlinear control, distributed parameter systems, hybrid communication network simulation and management. $\mathrm{He}$ is currently serving on the editorial boards of Mathematics of Control, Signals, and Systems, of Systems and Control: Foundations and Applications, of IMA Journal of Mathematical Control and Information, Systems Automation-Research Applications, and he is the Managing Editor of the series Progress in Automation and Information Systems from Springer-Verlag. He has numerous publications in control and communication systems, and is the co-editor of Recent Progress in Stochastic Calculus (New York: Springer-Verlag, 1990).

Dr. Baras has won several awards in his career. Among these are a 1978 Naval Research Laboratory Research Publication Award, the 1980 Outstanding Paper Award of the IEEE Control Systems Society, and the 1983 and 1993 Alan Berman Research Publication Awards from the Naval Research Laboratory. He has served on the IEEE Engineering Research and Development Committee, the Aerospace Industries Association Advisory Committee on Advanced Sensors, the IEEE Fellow Evaluation Committee, and the IEEE Control Systems Society Board of Governors (1991-1993). He is a member of Sigma Xi, the American Mathematical Society, and the Society for Industrial and Applied Mathematics.

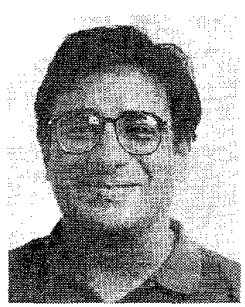

Nital S. Patel (S'91-M'96) received the B Tech. degree from the Indian Institute of Technology, Kanpur, India, in 1991, and the M.S. and Ph.D. degrees from the University of Maryland, College Park, in 1993 and 1995, respectively, all in electrical engineering.

Currently, he is a Research Associate at the Institute for Systems Research. His research interests include robust nonlinear control, intelligent control, modeling, numerical methods for large scale stochastic control problems, and their applications to control and coordination of manufacturing processes. 